\title{
Effect of Electroacupuncture on Visceral Hyperalgesia, Serotonin and Fos Expression in an Animal Model of Irritable Bowel Syndrome
}

\author{
Justin $C Y$ Wu, MD ${ }^{1 *}$, Eric TC Ziea, $M D^{1}$, Lixing Lao, $M D^{3}$, Emma FC Lam, MD ${ }^{1}$, Catherine SM Chan, $M D^{1}$, \\ Angela YQ Liang, MD ${ }^{1}$, Sunny LH Chu, MD ${ }^{1}$, David TW Yew, MD ${ }^{2}$, Brian M. Berman, MD ${ }^{3}$ and Joseph JY Sung ${ }^{1}$ \\ ${ }^{1}$ Institute of Digestive Disease, The Chinese University of Hong Kong, ${ }^{2}$ Department of Anatomy, The Chinese University of Hong Kong, Hong \\ Kong, ${ }^{3}$ Center for Integrative Medicine, University of Maryland School of Medicine, Baltimore, Maryland, USA
}

\begin{abstract}
Background/Aims
While it is well established that acupuncture relieves somatic pain, its therapeutic effect on visceral pain such as irritable bowel syndrome (IBS) is unclear. We evaluated the effect of acupuncture in treating visceral hyperalgesia in an animal model.

\section{Methods}

Sprague-Dawley rats ( $n=8$ per group) with prior neonatal maternal separation stress were randomly allocated to receive 3-day treatment of either electroacupuncture (EA) or sham acupuncture at acupoint ST-36. Another group of rats without prior maternal separation was included as non-handled controls. Colorectal distension was performed on the day after acupuncture treatment. The 3 groups were compared for pain threshold as determined by abdominal withdrawal reflex and visceromotor response as measured by electromyogram. Colon, spinal cord, and brainstem were sampled for topographic distribution and quantitative assessment of serotonin and Fos expression by immunohistochemistry.
\end{abstract}

\section{Results}

Rats in EA group had significantly higher pain threshold compared to those in sham acpuncture group $(25.0 \pm 5.7 \mathrm{mmHg}$ vs $18.7 \pm 5.2 \mathrm{mmHg}, p=0.01)$ and it was comparable with that of non-handled treatment naïve controls $(29.4 \pm 9.3 \mathrm{mmHg}$, $p=0.28$. They also had lower visceromotor response as measured by electromyogram compared to those received sham acupuncture at all colorectal distension pressures. EA significantly suppressed Fos expression in doral raphe nuclei of brainstem, superficial dorsal horn of spinal cord and colonic epithelium but suppressed 5-HT expression only in brainstem and spinal cord.

\section{Conclusions}

Electro acupuncture attenuates visceral hyperlagesia through down-regulation of central serotonergic activities in the brain-gut axis.

(J Neurogastroenterol Motil 2010;16:306-314)

Key Words

Electroacupuncture, Hyperalgesia, Brain-gut axis, Fos, Serotonin

Received: May 21, 2010 Revised: July 14, 2010 Accepted: July 15, 2010

(c) This is an Open Access article distributed under the terms of the Creative Commons Attribution Non-Commercial License (http://creativecommons. org/licenses/by-nc/3.0) which permits unrestricted non-commercial use, distribution, and reproduction in any medium, provided the original work is properly cited.

*Correspondence: Justin CY Wu, MD

Department of Medicine and Therapeutics, Prince of Wales Hospital, The Chinese University of Hong Kong, Shatin , Hong Kong Tel: +852-2632-3593, Fax: +852-2637-3852, E-mail: justinwu@cuhk.edu.hk

Financial support: This study was supported by the National Center for Complementary and Alternative Medicine (NCCAM) at the National Institutes of Health (NIH), grant number 1-U19-AT003266-01 (PI: Brian Berman, University of Maryland Baltimore), research funds of Department of Medicine \& Therapeutics and Li Ka Shing Insitute of Health Sciences, The Chinese University of Hong Kong.

Conflicts of interest: None. 


\section{Introduction}

Irritable bowel syndrome (IBS) is a common functional bowel disorder that is characterized by recurrent abdominal pain and disturbed bowel movements with the absence of demonstrable pathology. Albeit a benign condition, IBS is associated with significant morbidity. ${ }^{1}$ While the pathophysiology of IBS is still poorly understood, it is generally accepted that visceral hyperalgesia plays an important role. ${ }^{2,3}$ It has been postulated that visceral hyperalgesia is attributed to abnormalities in perception, transmission and processing of visceral nociceptive signals in the brain-gut axis. $^{4-8}$

Several neurotransmitters have been implicated in the development of visceral hyperalgesia. Amongst them, serotonin (5-HT) hyperactivity has been reported to be associated with visceral hypersensitivity in IBS patients. ${ }^{9,10}$ The role of 5-HT hyperactivity in IBS was further supported by the effectiveness of $5-\mathrm{HT}_{3}$ receptor antagonist for treatment of IBS. ${ }^{11}$ It has been shown that stress in early life contributed to visceral hyperalgesia and subsequent development of IBS in adulthood, and it is probably associated with 5-HT hyperactivity in brain-gut axis. ${ }^{12}$

Acupuncture has been found to be useful in treatment of various somatic pain and inflammatory conditions. ${ }^{13}$ The anti-hyperalgesic effect of acupuncture is mainly mediated through activation of descending inhibitory system including opioidergic, adrenergic and serotonergic pathways at both central and peripheral nervous systems. ${ }^{14-19}$ Acupuncture has also been shown to modulate motility and secretory functions of gastrointestinal tract. ${ }^{20,21}$ However, the role of acupuncture in treatment of visceral pain such as IBS is less defined. Although some uncontrolled studies reported increased rectal sensory thresholds and improvement in bowel symptoms in IBS patients treated with acupuncture, ${ }^{22,23}$ these results were not supported in sham controlled study. ${ }^{24}$ It has been shown that most acupuncture trials for IBS were of poor quality and effect of acupuncture is primarily a placebo response. ${ }^{25-27}$ The objective of this study was to evaluate the effect of electroacupuncture on visceral pain perception and 5-HT expression in brain-gut axis in a central early stress model of visceral hyperalgesia.

\section{Materials and Methods}

\section{Animal model of visceral hyperalgesia}

A rat model of visceral hyperalgesia was developed using ne- onatal maternal separation stress (NMSS) as described by Coutinho et $\mathrm{al}^{28}$ This was an early life stress model targeted to central nervous system, which induced visceral hyperalgesia in adulthood. Visceral hyperalgesia induced by NMSS was associated with increased serotonergic activities in both central and enteric nervous systems. ${ }^{12}$ The observations of enhanced anxiety-like behaviors, alterations in serotonergic responsiveness, stress-induced visceral hyperalgesia in this model fulfilled both face and construct validity as an animal model of IBS.

Pregnant female Sprague-Dawley rats were purchased from Laboratory Animal Services Center, The Chinese University of Hong Kong. Litters of surviving male pups were randomly housed with one lactating dam per cage and each litter comprising no more than 10 neonates, on a 12:12-hour light-dark cycle (lights on at $7 \mathrm{AM}$ ) at room temperature $20^{\circ} \mathrm{C}$ until they were 22 days old. The dams had access to food and water ad labium. Litters were weaned on postnatal day 22 and housed in groups of 8 through postnatal day 60. Neonatal maternal separation (MS) was performed during the light component of the cycle. Litters were exposed to a 180-minute period of daily maternal separation on postnatal day 2-21 inclusive. Manipulation of the litters commenced at 0800 hours: dams were removed to separation cages, while each litter was removed as a group to an adjacent room. At the end of the separation period, litters were reunited with their dams in the maternity cages. Another group of rats was not treated with neonatal maternal separation and they served as non-handled controls $(\mathrm{NH})$. The protocols were approved by Institutional Animal Care and Use Committee of University of Maryland, Baltimore, and the Animal Ethics Committee of the Chinese University of Hong Kong. Animal care and experimental procedures were conducted in accordance with institutional ethics guidelines.

\section{Electromyogram}

Electromyogram (EMG) electrode implantation was conducted as previously reported. ${ }^{29}$ On postnatal day 50 , the rats were anaesthetized and a pair of non-insulated tips of electrodes (Teflon coated stainless steel wire, Cooner Wire Sales, Chatsworth, CA, USA) was stitched in parallel, $5 \mathrm{~mm}$ apart, superior to the inguinal ligament, into the external oblique muscle. The electrodes were threaded subcutaneously to emerge at the back of the neck, where they were secured by a 2-O silk suture. Post-operative wound pain was treated with subcutaneous administration of $0.5 \%$ lidocaine and povidone-iodine was applied to the surface of sutured wound to prevent infection. After the procedure, rats 
were housed individually.

\section{Electroacupuncture}

On postnatal day 57 (7 days after EMG electrode implantation), the MS rats were randomly allocated to one of the following acupuncture groups: Real electroacupuncture (EA) or sham acupuncture (SA). NH control rats were not given any acupuncture treatment. A modified electroacupuncture technique reported by Lao and Tabosa was used. ${ }^{30}$ Stainless steel acupuncture needles (gauge \#32, $2.5 \mathrm{~cm}$ in length) were inserted bilaterally at a depth of $7 \mathrm{~mm}$ into the two acupoints of Zusanli (Stomach 36 or ST-36), which were located at $5 \mathrm{~mm}$ lateral to the anterior tubercle of the tibia and $10 \mathrm{~mm}$ below the knee joint. The paired needles were connected to output terminals of an electroacupuncture apparatus (Cefar ${ }^{\circledR}$ Acus4, Cefar Medical AB, Sweden), and the electrical wires were affixed on the animal's body with adhesive tape. An electrical current of $10 \mathrm{~Hz}, 0.18 \mathrm{~ms}$ pulse width, was delivered for 20 minutes. Stimulation intensity was adjusted to a level that elicited a slight muscle twitch at the acupuncture site and was limited to maximum of $3 \mathrm{~mA}$ to minimize animal discomfort.

Sham acupuncture treatment was performed by inserting needles at the acupoints with no electrical stimulation or manual manipulation. During treatment, the un-anaesthetized rats were placed in a $5 \times 8$ inch transparent plastic chamber where they were unrestrained and allowed to mobilize. Acupuncture was administered once daily for 3 consecutive days in both EA and SA groups from postnatal day 57 to 59 .

\section{Graded colorectal balloon distension and measure- ment of visceromotor response}

Colorectal balloon distension was used for noxious mechanical stimulation of the colon. A balloon catheter was constructed from a latex glove finger ( $4 \mathrm{~cm}$ of length) attached to an angiocatheter connected via a $\mathrm{Y}$ connector to a syringe pump and a sphygmomanometer. On postnatal day 60, animals were sedated with $2 \%$ isoflurane in oxygen (inhalation of $0.2 \mathrm{~L} / \mathrm{min}$ ) for 5 minutes. The balloon catheter was inserted into the distal colon 1 $\mathrm{cm}$ from the anal verge and secured to the proximal end of rat's tail with tape. The rats were then placed on an elevated platform and allowed to wake up and recover for at least 30 minutes. Phasic Colorectal balloon distension was comprised of 20-second inflation followed by 4-minute interval of deflation with stepwise increase in distension pressure $(10,20,40,60$ and $80 \mathrm{mmHg})$. Abdominal withdrawal reflex scores were assessed by a single in- dependent observer who was unaware of the group assignment. Colorectal balloon distension pain threshold was defined as the distension pressure that evoked a visually identifiable contraction of the abdominal wall. ${ }^{31}$ The measurements were repeated once and the average of the 2 measurements would be used for determination of pain threshold pressure.

Visceromotor responses to Colorectal balloon distension were measured quantitatively by EMG. EMG signals were obtained by an EMG signal receiver linked to a raw data analog converter (Model ML870 PowerLab 8/30, Bio-amplifier: ML132 Bio Amp, AD Instruments) at a rate of 2,000 samples per second using powerLab software (version 5, AD Instruments). Each 20 -second Colorectal balloon distension period and its preceding 20 -second interval of baseline recording were extracted from the original raw EMG data file. The extracted data was filtered with a bandpass filter (LP, $10 \mathrm{~Hz}$; HP, $500 \mathrm{~Hz}$ ) to correct for background and environmental noise, and artefacts caused by movement and breathing. Final data sets were obtained by subtracting the 20-second baseline readings from the 20-second Colorectal balloon distension readings. The area under curve (AUC) values were calculated with Matlab software.

\section{Immunohistochemistry}

Tissue harvesting was performed 1 hour after Colorectal balloon distension stimulation. The time lag allowed for deflating and removing balloon, dismantling syringe pump \& sphygmomanometer and administering general anesthesia before cardiac perfusion. The rats were sacrificed by intraperitoneal injection with $7 \%$ chlorohydrate $(35 \mathrm{mg} / 100 \mathrm{~g}$ body weight) and transcardial perfusion with $0.85 \%$ saline (about $100 \mathrm{~mL}$ ) followed by $4 \%$ paraformaldehyde in $0.1 \mathrm{~mol} / \mathrm{L}$ phosphate buffer $(\mathrm{pH} 7.4$, about $300 \mathrm{~mL}$ ). The tissues from distal colon (6 cm from anus), lumbosacral spinal cord (L6-S1) and brainstem (dorsal raphe nucleus [DRN]) were harvested for immunohistochemical assessment of Fos and 5-HT expression. They were post-fixed in paraformaldehyde overnight, cryoprotected in $25 \%$ sucrose in 0.1 $\mathrm{mol} / \mathrm{L}$ phosphate buffer for 48 hours, then embedded in OCT compound and frozen in iso-pantane chilled in liquid nitrogen. Sections ( $20 \mu \mathrm{m}$ for colon and $40 \mu \mathrm{m}$ for spinal cord and brain) were frozen at $-20^{\circ} \mathrm{C}$. Fos, a protein encoded by Fos pro-oncogene, was used as a biomarker for neuronal activation in response to noxious stimuli such as colorectal distension or inflammation. ${ }^{30,32,33}$ In intestinal epithelium, Fos also served as marker of mast cell or inflammatory cell activation. . $^{3,35}$

Tissues sections were rinsed 3 times for 10 minutes each in 
$0.01 \mathrm{~mol} / \mathrm{L}$ phosphate-buffered saline (PBS, $\mathrm{pH} 7.4$ ), pre-incubated for 20 minutes with $0.3 \% \mathrm{H}_{2} \mathrm{O}_{2}$ in $0.01 \mathrm{~mol} / \mathrm{L} \mathrm{PBS}$, then pre-incubated for 30 minutes with $5 \%$ normal goat serum (NGS) in $0.01 \mathrm{~mol} / \mathrm{L} \mathrm{PBS}$ and incubated overnight at room temperature with rabbit anti-Fos polyclonal antibody (rabbit anti-rat, 1:16,000, Oncogene Research Products, San Diego, CA, USA) in $0.01 \mathrm{~mol} / \mathrm{L}$ PBS with $1 \%$ NGS and $0.3 \%$ Triton $\mathrm{X}-100$. After rinsing, sections were incubated for 90 minutes with secondary goat anti-rabbit immunoglobulin $G(1: 200$, Vectastain ABC kit, Vector Laboratories, Burlingame, CA, USA) in $0.01 \mathrm{~mol} / \mathrm{L} \mathrm{PBS}$ with $1 \% \mathrm{NGS}$ and $0.3 \%$ Triton, rinsed 3 times for 10 minutes each in $0.01 \mathrm{~mol} / \mathrm{L} \mathrm{PBS}$, incubated for 90 minutes with tertiary antibody $(1: 100$, avidin DH/biotinylated horseradish peroxidase $\mathrm{H}$ complex, Vectastain ABC kit, Vector Laboratories) in $0.01 \mathrm{~mol} / \mathrm{L} \mathrm{PBS}$ and rinsed $2 \times 10$ minutes in $0.01 \mathrm{~mol} / \mathrm{L}$ PBS. A peroxidase reaction was performed to visualize Fos immunolabeling by incubating with $0.05 \%$ 3,3-diaminobenzidine tetrahydrochloride and $0.01 \% \mathrm{H}_{2} \mathrm{O}_{2}$ for $5 \mathrm{mi}-$ nutes before decanting and stopping the reaction with $0.01 \mathrm{~mol} / \mathrm{L}$ PBS. To assess antibody specificity, incubation with the primary antibody was omitted for some sections and no significant staining was observed in this case.

Similarly, tissues were washed in $0.1 \mathrm{~mol} / \mathrm{L}$ PBS, incubated with a 5-HT polyclonal antibody (TX rabbit anti-rat, $1: 1,000$, Alpha Diagnostic International, Inc, San Antonio, TX, USA) for 12 hours, followed by a 2-hour incubation of goat anti-rabbit IgG conjugated with fluorescein isothiocyanate (1:200, Jackson Immuno Research, West Grove, PA, USA) at room temperature. Primary and secondary antibodies were diluted in $0.3 \%$ Triton X-100 (in $0.1 \mathrm{~mol} / \mathrm{L}$ PBS). NGS (Vectastain ABC kit, Vector Laboratories) was added to a final concentration of $10 \%$ to reduce the non-specific background staining.

\section{Image analysis}

All stained sections of harvested tissue were examined using a Zeiss Axiophot 2 imaging Universal Fluorescence Photo-Microscope with Axio cam (Universal Microscope, Germany). Images were captured by Spot Cooled Color Digital Camera equipped with Spot32 software (Diagnostic Instrument, Sterling Heights, MI, USA). All images were analyzed by Metamorph 4.0 software. This software performed automated measurements through an interactive threshold editing function. Well-stained nuclei of Fos or 5-HT were chosen as reference. The computer recognized these as the standard grayscale level, grouped all the contiguous pixels that fell within that level into "object" and seg- regated them from the background. Since the pixel density of the background was either lower or higher than "object", any area of the slide equivalent to the standard grayscale was marked as object and designated as the threshold area. In addition, any area lower than or beyond the standard grayscale was marked as background. The percentage of threshold area was calculated as threshold area of the selected area divided by total selected area $\mathrm{X}$ $100 \%$.

Serotonin and Fos expression were quantified in the colonic mucosa at $6 \mathrm{~cm}$ above anus, superficial dorsal horn (SDH) of spinal cord (lamina I \& II) and DRN. Five sections of each tissue were prepared in random fashion for image capture at different magnification (colon, $\times 200$; spinal cord, $\times 400$; and brain, $\times$ 100). After interactive thresholding, 5 areas were randomly selected for measurement by the image analysis system. All imaging procedures were performed by a single investigator who was unaware of the grouping of rats.

\section{Statistical methods}

All data were presented as means \pm SE. Statistical analyses were performed by using SPSS11.0 (SPSS Inc, Chicago, IL, USA). Comparisons between groups were made using unpaired $t$ test, one-way ANOVA and Mann-Whitney $\mathrm{U}$ test as appropriate. Differences with two-tailed $\mathrm{p}<0.05$ were considered statistically significant. Bonferroni correction was performed for post-hoc analyses.

\section{Results}

\section{Effect of electroacupuncture on pain threshold and visceromotor response}

Twenty-four rats were randomly allocated into 3 groups ( $\mathrm{n}=$ 8 per group): (1) MS rats with real EA, (2) MS rats with SA and (3) NH treatment naïve rats. Comparisons of mean pain threshold pressures among the 3 groups were shown in Figure 1A. Rats in EA and $\mathrm{NH}$ group had significantly higher pain threshold pressures in response to Colorectal balloon distension than those in SA group (EA vs SA, $25.0 \pm 5.7 \mathrm{mmHg}$ vs 18.7 $\pm 5.2 \mathrm{mmHg}, \mathrm{p}=0.01 ; \mathrm{NH}$ vs $\mathrm{SA}, 29.4 \pm 9.3 \mathrm{mmHg}$ vs 18.7 $\pm 5.2 \mathrm{mmHg}, \mathrm{p}=0.02$ ). There was no significant difference in pain threshold pressures between EA and NH rats ( $p=0.28)$.

Visceromotor responses to graded Colorectal balloon distension at different distension pressures as measured by EMG were shown in Figure 1B. Rats in EA group had significantly 
A

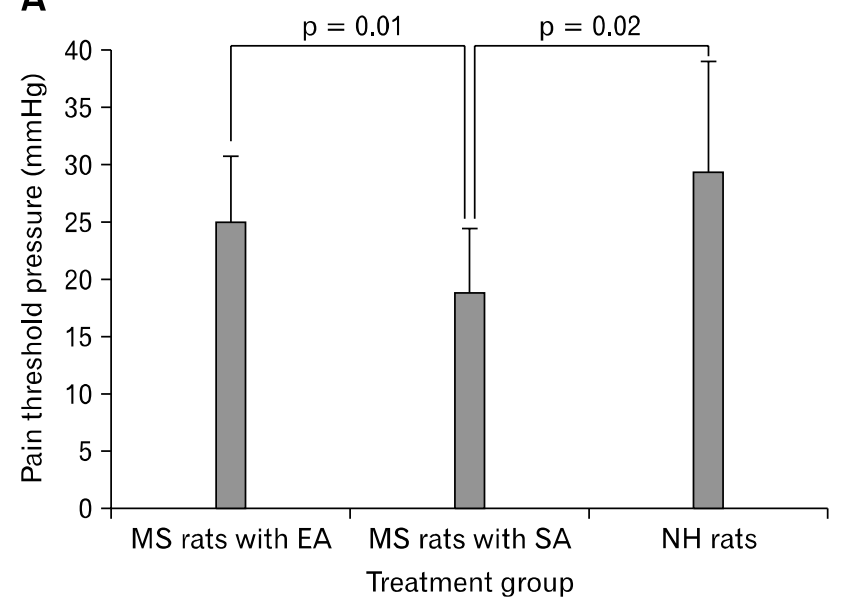

B

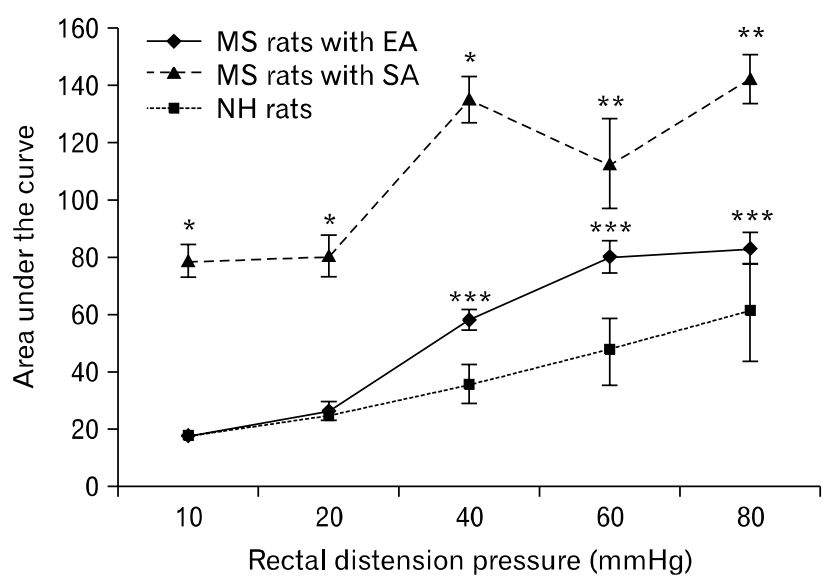

Figure 1. Effect of electroacupuncture on pain threshold and visceromotor response. (A) Rats in electro acupuncture (EA) group and non-handled control (NH) group had significantly higher pain threshold pressures in response to colorectal balloon distension than those in sham acupuncture (SA) group. (B) Rats in SA group had significantly higher mean area under curve values compared to those in EA and NH group at all distension pressures. MS, maternal separation. Data were presented as mean \pm SEM. Two-tailed p-values were determined by one-way ANOVA. ${ }^{*} p<0.001,{ }^{*}$ p $<$ 0.01 for post hoc comparisons of SA to EA and $\mathrm{NH}$ group. ${ }^{* * *} \mathrm{p}<0.05$ for post hoc comparisons between EA and $\mathrm{NH}$ group using Bonferroni correction.
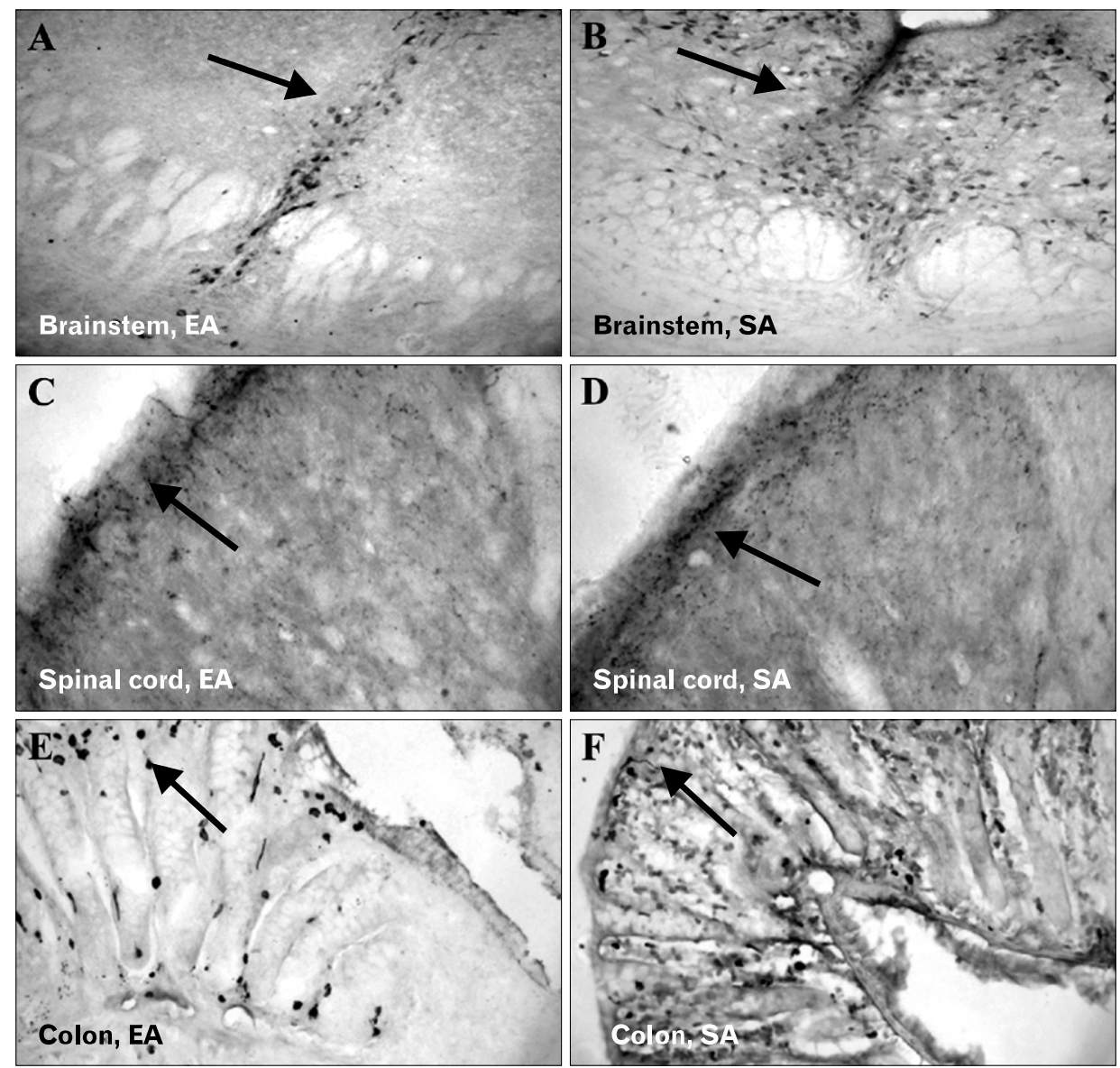

Figure 2. Comparison of serotonin (5-HT) expression between electroacupuncture (EA) and sham acupuncture (SA) groups. Immunohistochemistry showed significantly lower 5-HT expression (arrowed) in the dorsal raphe nucleus of the brain (A, EA; B, SA; × 40) and laminae I \& II of superficial dorsal horn of the spinal cord $(\mathrm{C}, \mathrm{EA} ; \mathrm{D}, \mathrm{SA} ; \times 40)$ in rats treated with EA compared to those treated with SA. There was no difference in 5-HT expression at colonic mucosa between the 2 groups (E, EA; F, SA; ×40). 
lower AUC values compared to those in SA group at all distension pressures $(10 \mathrm{mmHg}, \mathrm{p}<0.001 ; 20 \mathrm{mmHg}, \mathrm{p}<0.001$; $40 \mathrm{mmHg}, \mathrm{p}<0.001 ; 60 \mathrm{mmHg}, \mathrm{p}=0.003 ; 80 \mathrm{mmHg}, \mathrm{p}=$ 0.004). Furthermore, the AUC values of SA group were also significantly higher than those of $\mathrm{NH}$ group at all distension pressures (10 mmHg, p < 0.001; $20 \mathrm{mmHg}$, p $<0.001 ; 40 \mathrm{mmH}$,: $\mathrm{p}<0.001 ; 60 \mathrm{mmHg}, \mathrm{p}=0.002 ; 80 \mathrm{mmHg}, \mathrm{p}<0.001)$. There was no significant difference in AUC values at low distension pressures between EA and $\mathrm{NH}$ groups but rats in EA group had significantly higher AUC values than those in $\mathrm{NH}$ group at higher distension pressures $(10 \mathrm{mmHg}, \mathrm{p}=0.82 ; 20$ $\mathrm{mmHg}, \mathrm{p}=0.64 ; 40 \mathrm{mmHg}, \mathrm{p}=0.01 ; 60 \mathrm{mmHg}, \mathrm{p}=0.01$; $80 \mathrm{mmHg}, \mathrm{p}=0.03$ ).

\section{Effect of electroacupuncture on serotonin and Fos expression in brain-gut axis}

The serotonin immunoreactivities at different regions were presented in Figure 2. Compared to rats in SA group, rats in EA

Table 1. Comparison of Median Threshold Area Percentages of 5-HT Expression at Dorsal Raphe Nucleus of Brainstem, Superficial Dorsal Horn of Spinal Cord and Colonic Mucosa among Rats of Electroacupuncture, Sham Acupuncture and Non-handled Control Groups

\begin{tabular}{|c|c|c|c|}
\hline & $\mathrm{EA}(\mathrm{n}=8)$ & $\mathrm{SA}(\mathrm{n}=8)$ & $\mathrm{NH}(\mathrm{n}=8)$ \\
\hline DRN of brainstem (median [range]) & $0.25^{\mathrm{a}}(0.01-0.54)$ & $1.56^{\mathrm{b}}(0.01-4.60)$ & $0.14(0.01-0.68)$ \\
\hline SDH of spinal cord (median [range]) & $0.61^{\mathrm{c}}(0.19-1.59)$ & $3.23^{\mathrm{d}}(1.82-4.43)$ & $0.29(0.11-0.87)$ \\
\hline Colonic mucosa (median [range]) & $1.73^{\mathrm{e}}(0.34-6.73)$ & $2.58^{\mathrm{f}}(0.5-8.78)$ & $1.26(0.21-3.21)$ \\
\hline
\end{tabular}

EA, electroacupuncture; SA, sham acupuncture; NH, non-handled control; DRN, dorsal raphe nucleus; SDH, superficial dorsal horn.

Median values (range) were compared using Mann-Whitney $\mathrm{U}$ test. Comparisons between EA and SA group $\left({ }^{\mathrm{a}} \mathrm{p}=0.02\right)$ and between SA and NH group $\left({ }^{b} \mathrm{p}=0.006\right)$ at DRN of brainstem, comparisons between EA and SA group ( $\mathrm{c} p=0.001)$ and between SA and NH group $(\mathrm{p}<0.001)$ at SDH of spinal cord, comparisons between EA and SA group $\left({ }^{e} p=0.14\right)$ and between $\mathrm{SA}$ and $\mathrm{NH}$ group $\left({ }_{\mathrm{f}}^{\mathrm{f}}=0.01\right)$ at colonic mucosa.
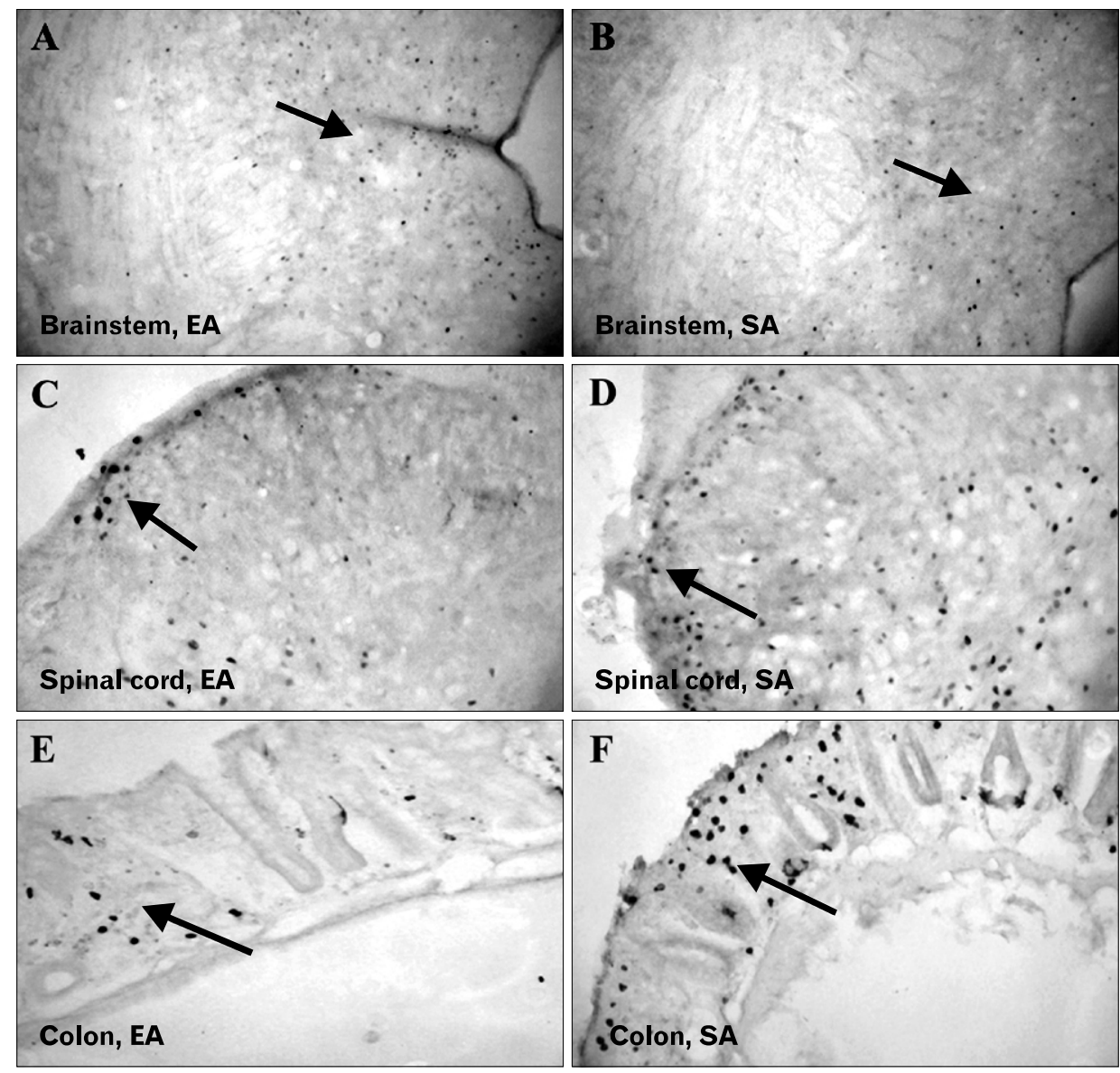

Figure 3. Comparison of Fos expression between electroacupuncture (EA) and sham acupuncture (SA) groups. Immunohistochemistry showed significantly lower Fos expression (arrowed) in the dorsal raphe nucleus of the brain (A, EA; B, SA; × 40), laminae I and II of superficial dorsal horn of the spinal cord (C, EA; D, SA; × 40) and colonic mucosa $(\mathrm{E}, \mathrm{EA} ; \mathrm{F}, \mathrm{SA} ; \times 40)$ in rats treated with EA compared to those treated with SA. Fos positive cells were highly abundant on colonic epithelium among $\mathrm{SA}$ rats compared to $\mathrm{EA}$ rats. 
Table 2. Comparison of Median Threshold Area Percentages of Fos Expression at Dorsal Raphe Nucleus of Brainstem, Superficial Dorsal Horn of Spinal Cord and Colonic Mucosa among Rats of Electroacupuncture, Sham Acupuncture and Non-handled Control Groups

\begin{tabular}{lccc}
\hline & EA $(\mathrm{n}=8)$ & SA $(\mathrm{n}=8)$ & NH $(\mathrm{n}=8)$ \\
\hline DRN of brainstem (median [range]) & $0.11^{\mathrm{a}}(0.02-0.59)$ & $0.76^{\mathrm{b}}(0.06-1.23)$ & $0.16(0.03-0.72)$ \\
SDH of spinal cord (median [range]) & $0.23^{\mathrm{c}}(0.10-0.60)$ & $1.84^{\mathrm{d}}(0.51-3.82)$ & $0.19(0.06-0.42)$ \\
Colonic mucosa (median [range]) & $1.92^{\mathrm{e}}(0.08-3.05)$ & $8.42^{\mathrm{f}}(1.82-12.02)$ & $2.34(0.02-4.22)$ \\
\hline
\end{tabular}

EA, electroacupuncture; SA, sham acupuncture; $\mathrm{NH}$, non-handled control; DRN, dorsal raphe nucleus; SDH, superficial dorsal horn.

Median values (range) were compared using Mann-Whitney $\mathrm{U}$ test. Comparisons between EA and SA group $\left({ }^{\mathrm{a}} \mathrm{p}=0.01\right)$ and between SA and $\mathrm{NH}$ group $\left({ }^{\mathrm{b}} \mathrm{p}=0.02\right)$ at DRN of brainstem, Comparisons between EA and SA group ( $\left.{ }^{c} \mathrm{p}<0.001\right)$ and between SA and NH group $\left({ }^{d} \mathrm{p}<0.001\right)$ at SDH of spinal cord, comparisons between EA and SA group $\left({ }^{e} \mathrm{p}=0.005\right)$ and between $\mathrm{SA}$ and $\mathrm{NH}$ group $\left({ }_{\mathrm{f}}^{\mathrm{f}}=0.01\right)$ at colonic mucosa.

group had significantly lower 5-HT expression in DRN ( $\mathrm{p}=$ $0.02)$ and SDH of spinal cord $(p=0.001)$ but not in colonic mucosa $(p=0.14)$ (Table 1). Rats in SA group also had significantly higher 5-HT expression at all the 3 regions compared to those in $\mathrm{NH}$ groups. There was no significant difference in 5-HT expression between EA and NH groups.

The Fos immunoreactivities at different regions were presented in Figure 3. Compared to rats in SA group, rats in EA group had significantly lower Fos expression in DRN ( $p=0.01)$, SDH of spinal cord $(\mathrm{p}<0.001)$ and colonic mucosa $(\mathrm{p}=0.005)$ (Table 2). Fos positive cells were highly abundant on colonic epithelium among SA compared to EA rats. Rats in SA group also had significantly higher Fos expression at all the 3 regions compared to NH groups. There was no significant difference in Fos expression between EA and $\mathrm{NH}$ groups.

\section{Discussion}

We set out to evaluate the effect and mechanism of electroacupuncture for treatment of IBS in a neonatal maternal separation stress model of visceral hyperalgesia. We found that electroacupuncture applied to acupoint ST-36 significantly increased pain threshold and decreased visceromotor response to graded Colorectal balloon distension. The attenuation of visceral pain perception was associated with decreased expression of 5-HT primarily at $\mathrm{DRN}$ and $\mathrm{SDH}$ of spinal cord but not at colonic mucosa.

There were a few studies reporting the visceral anti-hyperalgesic effect of electroacupuncture using different animal models of visceral hyperalgesia. Using a neonatal colonic irritation model, Cui et $\mathrm{al}^{36}$ reported that electroacupuncture at ST-36 significantly suppressed abdominal withdrawal reflex score and magnitude of EMG in response to Colorectal balloon distension compared to sham treatment group. Tian et $\mathrm{al}^{19}$ further reported that electroacupuncture induced both attenuation of visceral hyperalgesia and stress-induced colonic motor dysfunction, which was associated with decreased 5-HT concentration and increased 5-HT transporter expression in colonic tissue. However, there was no sham control group in this study. Other studies have shown that electroacupuncture also reduced pain via activation of central opioid pathway and down-regulation of spinal cord NMDA (N-methyl-D-aspartate) receptors. ${ }^{37,38}$ Yet, there is still a lack of sham-controlled study that evaluates the role of central serotonergic pathway in electroacupuncture induced visceral anti-hyperalgesia.

In this study, we studied the effects of electroacupuncture on both peripheral and central serotonergic activities in a sham-controlled manner. There has been concern about the appropriateness of the use of invasive sham, which involved needle insertion at the acupoints, because of probable non-specific analgesic effects through diffuse noxious inhibitory controls (DNIC). ${ }^{39} \mathrm{We}$ employed invasive sham because it enabled better blinding of investigators. Moreover, any observed difference between real electroacupuncture and sham treatment would be attributed to genuine effects of electroacupuncture rather than DNIC.

Our study showed that electroacupuncture significantly reduced visceromotor response to Colorectal balloon distension compared to sham acupuncture. This finding is consistent with the anti-hyperalgesic effects observed in previous studies. ${ }^{19}$ Furthermore, we observed that the pain reduction was associated with significant reduction in serotonergic activities and Fos expression. The reduction in 5-HT expression was primarily seen at spinal cord and brainstem. The role of central serotonergic activities in modulation of visceral sensation is poorly understood. The SDH of the spinal cord (laminae I and II) receives input signals from both primary afferents of peripheral organs and serotonergic projections from the brainstem. We have previously reported that neonatal maternal separation stress led to visceral hyperalgesia, which was associated with increased serotonergic activities at $\mathrm{SDH}$ of spinal cord and dorsal raphe nucleus. ${ }^{12}$ On the other hand, serotonergic depletion of the lum- 
bar spinal cord with 5,7-DHT (5,7 di-hydroxytryptamine) creatinine sulphate reduced expression of transcription factors for dorsal horn neurone sensitization (eg, Fos and Methyl-CpGbinding protein 2) and significantly attenuated mechanical sensitivity after injection of Complete Freund's Adjuvant. ${ }^{40}$ These findings suggested that heightened serotonergic activities at $\mathrm{SDH}$ of spinal cord and DRN are responsible for visceral hyperalgesia. On the other hand, it has been shown that acupuncture suppresses Fos expression at spinal cord level through descending inhibitory pathway. ${ }^{41}$ Acupuncture inhibited Colorectal balloon distension-induced neuronal activity in the $\mathrm{SDH}$ and the inhibitory effect was absent in spinalized rats. ${ }^{42}$ It has also been reported that electroacupuncture inhibited gastric ulcer-induced Fos expression in the nucleus of the solitary tract in the brain. ${ }^{43}$ We postulate that electro-acupuncture at acupoint ST-36 down-regulates descending central serotonergic activities particularly at SDH of spinal cord and DRN, which results in reduction in transcription factors for spinal cord pain sensitization and hence induces visceral anti-hyperalgesic effect.

Another intriguing finding of our study was increased number of Fos positive cells at colonic mucosa in MS rats treated with sham acupuncture and it was reduced by electro-acupuncture. While Fos has been regarded as a marker for neuronal activation, it has also been associated with epithelial cell proliferation, mast cell degranulation and production of proinflammatory cytokines in activated lymphocyte in response to extracellular signals. The observation of increased number of Fos positive cells in colonic epithelium of sham-treated MS rats suggested that early stress on central nervous system may lead to abnormalities in gut mucosal proliferation or mast cell function. And these abnormalities may be reversed with electroacupuncture. Further studies are required to evaluate the role of central serotonergic pathways in regulation of mucosal proliferative and immune functions.

Our findings are somewhat different from previous study, which reported significant reduction in colonic expression of 5-HT with electroacupuncture in a gut-targeted rat model of neonatal colorectal mechanical irritation. ${ }^{19}$ This discrepancy may be attributed to different animal models employed. However, both studies lend support to the notion that electroacupuncture attenuates peripheral and central serotonergic activities rendered abnormal in early life, depending on the site of insults. Further studies are required to evaluate how electroacupuncture modulates serotonergic activity in brain-gut axis and its differential mechanisms involved in central and peripheral models of visceral hyperalgesia.

In conclusion, electroacupuncture attenuates visceral hyper- algesia in a central rat model of IBS. The anti-hyperalgesic effect is probably mediated by down-regulation of serotonergic activities in central nervous system. These findings provide theoretical basis supporting the use of acupuncture and modulation of central serotonergic system for treatment of IBS.

\section{Acknowledgements}

Its contents are solely the responsibility of the authors and do not necessarily represent the official views of NCCAM. The authors acknowledge all members of this international collaborative project for their collaborative efforts.

\section{References}

1. Halder SL, Locke GR III, Talley NJ, Fett SL, Zinsmeister AR, Melton LJ III. Impact of functional gastrointestinal disorders on health-related quality of life: a population-based case-control study. Aliment Pharmacol Ther 2004;19:233-242.

2. Mayer EA, Collins SM. Evolving pathophysiologic models of functional gastrointestinal disorders. Gastroenterology 2002;122:20322048.

3. Delvaux M. Role of visceral sensitivity in the pathophysiology of irritable bowel syndrome. Gut 2002;51(suppl 1):i67-i71.

4. Mertz H, Morgan V, Tanner G, et al. Regional cerebral activation in irritable bowel syndrome and control subjects with painful and nonpainful rectal distention. Gastroenterology 2000;118:842-848.

5. Lawal A, Kern M, Sidhu H, Hofmann C, Shaker R. Novel evidence for hypersensitivity of visceral sensory neural circuitry in irritable bowel syndrome patients. Gastroenterology 2006;130:26-33.

6. Naliboff BD, Berman S, Suyenobu B, et al. Longitudinal change in perceptual and brain activation response to visceral stimuli in irritable bowel syndrome patients. Gastroenterology 2006;131:352-365.

7. Aerssens J, Hillsley K, Peeters PJ, et al. Alterations in the brain-gut axis underlying visceral chemosensitivity in Nippostrongylus brasiliensis-infected mice. Gastroenterology 2007;132:1375-1387.

8. Anand P, Aziz Q, Willert R, Van Oudenhove L. Peripheral and central mechanisms of visceral sensitization in man. Neurogastroenterol Motil 2007;19(suppl 1):29-46.

9. Bearcroft CP, Perrett D, Farthing MJ. Postprandial plasma 5-hydroxytryptamine in diarrhoea predominant irritable bowel syndrome: a pilot study. Gut 1998;42:42-46.

10. Kerckhoffs AP, Ter Linde JJ, Akkermans LM, Samsom M. Trypsinogen IV, serotonin transporter transcript levels and serotonin content are increased in small intestine of irritable bowel syndrome patients. Neurogastroenterol Motil 2008;20:900-907.

11. Camilleri M, Northcutt AR, Kong S, Dukes GE, McSorley D, Mangel AW. Efficacy and safety of alosetron in women with irritable bowel syndrome: a randomised, placebo-controlled trial. Lancet 2000;355:1035-1040.

12. Ren $\mathrm{TH}, \mathrm{Wu} \mathrm{J}$, Yew $\mathrm{D}$, et al. Effects of neonatal maternal separation on neurochemical and sensory response to colonic distension in a rat 
model of irritable bowel syndrome. Am J Physiol Gastrointest Liver Physiol 2007;292:G849-G856.

13. Berman BM, Lao L, Langenberg P, Lee WL, Gilpin AM, Hochberg MC. Effectiveness of acupuncture as adjunctive therapy in osteoarthritis of the knee: a randomized, controlled trial. Ann Intern Med 2004;141:901-910.

14. Zhang RX, Lao L, Wang L, et al. Involvement of opioid receptors in electroacupuncture-produced anti-hyperalgesia in rats with peripheral inflammation. Brain Res 2004;1020:12-17.

15. Zhang RX, Wang L, Liu B, et al. Mu opioid receptor-containing neurons mediate electroacupuncture-produced anti-hyperalgesia in rats with hind paw inflammation. Brain Res 2005;1048:235-240.

16. Chang FC, Tsai HY, Yu MC, Yi PL, Lin JG. The central serotonergic system mediates the analgesic effect of electroacupuncture on ZUSANLI (ST36) acupoints. J Biomed Sci 2004;11:179-185.

17. Kim HW, Kwon YB, Han HJ, Yang IS, Beitz AJ, Lee JH. Antinociceptive mechanisms associated with diluted bee venom acupuncture (apipuncture) in the rat formalin test: involvement of descending adrenergic and serotonergic pathways. Pharmacol Res 2005;51:183-188.

18. Lee SH, Chung SH, Lee JS, et al. Effects of acupuncture on the 5 -hydroxytryptamine synthesis and tryptophan hydroxylase expression in the dorsal raphe of exercised rats. Neurosci Lett 2002; 332:17-20.

19. Tian XY, Bian ZX, Hu XG, Zhang XJ, Liu L, Zhang H. Electro-acupuncture attenuates stress-induced defecation in rats with chronic visceral hypersensitivity via serotonergic pathway. Brain Res 2006;1088:101-108.

20. Li Y, Tougas G, Chiverton SG, Hunt RH. The effect of acupuncture on gastrointestinal function and disorders. Am J Gastroenterol 1992;87:1372-1381.

21. Tougas G, Hunt RH. Relation of acupuncture and vagal gastric acid secretion. Gut 1995;36:800-801.

22. Chan J, Carr I, Mayberry JF. The role of acupuncture in the treatment of irritable bowel syndrome: a pilot study. Hepatogastroenterology 1997;44:1328-1330.

23. Xiao WB, Liu YL. Rectal hypersensitivity reduced by acupoint TENS in patients with diarrhea-predominant irritable bowel syndrome: a pilot study. Dig Dis Sci 2004;49:312-319.

24. Fireman Z, Segal A, Kopelman Y, Sternberg A, Carasso R. Acupuncture treatment for irritable bowel syndrome. A double-blind controlled study. Digestion 2001;64:100-103.

25. Röhrbock RB, Hammer J, Vogelsang H, Talley NJ, Hammer HF. Acupuncture has a placebo effect on rectal perception but not on distensibility and spatial summation: a study in health and IBS. Am J Gastroenterol 2004;99:1990-1997.

26. Schneider A, Enck P, Streitberger K, et al. Acupuncture treatment in irritable bowel syndrome. Gut 2006;55:649-654.

27. Lim B, Manheimer E, Lao L, et al. Acupuncture for treatment of irritable bowel syndrome. Cochrane Database Syst Rev 2006;(4): CD005111.

28. Coutinho SV, Plotsky PM, Sablad M, et al. Neonatal maternal separation alters stress-induced responses to viscerosomatic nociceptive stimuli in rat. Am J Physiol Gastrointest Liver Physiol 2002;282: G307-G316.

29. Traub RJ, Stitt S, Gebhart GF. Attenuation of c-Fos expression in the rat lumbosacral spinal cord by morphine or tramadol following noxious colorectal distention. Brain Res 1995;701:175-182.

30. Lao L, Zhang RX, Zhang G, Wang X, Berman BM, Ren K. A parametric study of electroacupuncture on persistent hyperalgesia and Fos protein expression in rats. Brain Res 2004;1020:18-29.

31. Al-Chaer ED, Kawasaki M, Pasricha PJ. A new model of chronic visceral hypersensitivity in adult rats induced by colon irritation during postnatal development. Gastroenterology 2000;119:1276-1285.

32. Kozlowski CM, Green A, Grundy D, Boissonade FM, Bountra C. The 5-HT(3) receptor antagonist alosetron inhibits the colorectal distention induced depressor response and spinal $c$-fos expression in the anaesthetised rat. Gut 2000;46:474-480.

33. Traub RJ, Tang B, Ji Y, Pandya S, Y fantis H, Sun Y. A rat model of chronic postinflammatory visceral pain induced by deoxycholic acid. Gastroenterology 2008;135:2075-2083.

34. Lee YN, Tuckerman J, Nechushtan H, Schutz G, Razin E, Angel P. c-Fos as a regulator of degranulation and cytokine production in FcepsilonRI-activated mast cells. J Immunol 2004;173:2571-2577.

35. Shima Y, Tajiri T, Taguchi T, Suita S. Increased expression of c-fos and c-jun in the rat small intestinal epithelium after ischemia-reperfusion injury: a possible correlation with the proliferation or apoptosis of intestinal epithelial cells. J Pediatr Surg 2006;41:830-836.

36. Cui KM, Li WM, Gao X, Chung K, Chung JM, Wu GC. Electro-acupuncture relieves chronic visceral hyperalgesia in rats. Neurosci Lett 2005;376:20-23.

37. Iwa M, Strickland C, Nakade Y, Pappas TN, Takahashi T Electroacupuncture reduces rectal distension-induced blood pressure changes in conscious dogs. Dig Dis Sci 2005;50:1264-1270.

38. Tian SL, Wang XY, Ding GH. Repeated electro-acupuncture attenuates chronic visceral hypersensitivity and spinal cord NMDA receptor phosphorylation in a rat irritable bowel syndrome model. Life Sci 2008;83:356-363.

39. Bing Z, Villanueva L, Le Bars D. Acupuncture and diffuse noxious inhibitory controls: naloxone-reversible depression of activities of trigeminal convergent neurons. Neuroscience 1990;37:809-818.

40. Géranton SM, Fratto V, Tochiki KK, Hunt SP. Descending serotonergic controls regulate inflammation-induced mechanical sensitivity and methyl-CpG-binding protein 2 phosphorylation in the rat superficial dorsal horn. Mol Pain 2008;4:35.

41. Li A, Wang Y, Xin J, et al. Electroacupuncture suppresses hyperalgesia and spinal Fos expression by activating the descending inhibitory system. Brain Res 2007;1186:171-179.

42. Rong PJ, Zhu B, Huang QF, Gao XY, Ben H, Li YH. Acupuncture inhibition on neuronal activity of spinal dorsal horn induced by noxious colorectal distention in rat. World J Gastroenterol 2005;11:1011-1017.

43. Wang H, Wang CY, Zhang JS, et al. Acupuncture therapy for experimental stomach ulcer and c-Fos expression in rats. World J Gastroenterol 2005;11:5517-5520. 